\title{
PATRONES DE PROCESAMIENTO ANTRÓPICO DE CAMÉLIDOS DURANTE LA TRANSICIÓN PLEISTOCENO- HOLOCENO EN CUEVA TÚNEL, MESETA CENTRAL DE SANTA CRUZ, ARGENTINA
}

\author{
ANTHROPIC CAMELID PROCESSING PATTERNS DURING THE PLEISTOCENE- \\ HOLOCENE TRANSITION IN CUEVA TÚNEL, CENTRAL PLATEAU OF SANTA \\ CRUZ, ARGENTINA
}

\author{
Catalina Valiza Davis ${ }^{1,2}$, Diego Damián Rindel ${ }^{1,2}$ y Rafael Sebastián Paunero ${ }^{3}$
}

\begin{abstract}
Este trabajo aborda el estudio de un conjunto óseo depositado en el sitio Cueva Túnel, Meseta Central de Santa Cruz, Argentina. El sitio abarca un rango temporal que va de fines del Pleistoceno hasta el Holoceno Tardío. Nos concentramos en su componente inferior, datado en ca.10500 años AP, que presenta una amplia diversidad de fauna, entre la que se incluyen varias especies hoy extintas. Se han identificado restos faunísticos correspondientes a los camélidos Lama guanicoe, Hemiauchenia paradoxa y Lama gracilis. El objetivo de este trabajo es analizar los patrones de consumo de los camélidos, dado que son los taxones que se hallan más representados en el conjunto analizado. Esta situación no es privativa de este sitio arqueológico, sino que se repite en otros conjuntos correspondientes a la transición Pleistoceno-Holoceno en Patagonia, indicando la importancia económica que los camélidos han tenido a lo largo del proceso de poblamiento de Patagonia. Sin embargo, no se han investigado en detalle las estrategias empleadas en el procesamiento y consumo de estos taxones, ni las diferencias intertaxonómicas en la explotación de estos recursos. Para cumplir con este objetivo, consideramos las siguientes variables: la asignación taxonómica, la frecuencia de partes esqueletarias, las evidencias de agentes y procesos naturales y el estudio de marcas de procesamiento y consumo humano. Estos datos son contextualizados con información acerca del ambiente ecológico, presentando una caracterización de los patrones de subsistencia desarrollados por los primeros grupos humanos que poblaron estos sectores.
\end{abstract}

Palabras claves: camélidos, procesamiento, consumo, Patagonia, Pleistoceno-Holoceno.

This paper aims to study a bone assemblage deposited in the Cueva Túnel site, located in the Central Plateau of Santa Cruz, Argentina. The site covers a temporal range that dates from late Pleistocene to Late Holocene. We focus on its lower component, dating back to ca. 10500 years BP, which presents a wide diversity of fauna, including several extinct species. Faunal remains have been found that correspond to three camelids species: Lama guanicoe, Hemiauchenia paradoxa and Lama gracilis. The objective of this paper, then, is to analyze the consumption patterns of camelids, as they are the most represented taxa in the analyzed assemblage. This situation is repeated in other sites corresponding to the Pleistocene-Holocene transition in southern Patagonia, indicating the economic importance that camelids have had throughout the initial Patagonia colonization process. However, the strategies employed in the processing and consumption of these taxa, and the inter-taxonomic differences in the exploitation of these resources have not been investigated in detail. To achieve this, we consider the following variables: taxonomic assignment, frequency of skeletal parts, evidence of taphonomic agents and the study of human processing and consumption marks. These data are contextualized with information about the ecological environment, in order to present a characterization of the subsistence and mobility patterns of the first human groups that populated the interior sectors of southern Patagonia.

Key words: Camelids, processing, consumption, Patagonia, Pleistocene-Holocene.

El estudio de los grupos de cazadores-recolectores correspondientes a momentos de la transición Pleistoceno-Holoceno en Patagonia ha sido abordado desde diferentes líneas de evidencia, tales como la tecnología lítica, el análisis de la organización y uso del espacio y el registro arqueofaunístico, entre otros (Borrero 2015; Borrero y Martin 2018; Cueto 2015; Frank 2012; Marchionni 2013; Méndez et al.

CONICET- Consejo Nacional de Investigaciones Científicas y Técnicas, Buenos Aires, Argentina.

División de Arqueología, Facultad de Ciencias Naturales y Museo, Universidad Nacional de La Plata, La Plata, Argentina. catavaliza@hotmail.com; rpaunero@fcnym.unlp.edu.ar

3 División Antropología, Facultad de Ciencias Naturales y Museo, Universidad Nacional de La Plata, La Plata, Argentina. drindelarqueo@yahoo.com 
2008; Miotti 1998 [1989]; Perez et al. 2016; Skarbun 2011, entre otros). Particularmente, el estudio de los restos faunísticos resulta fundamental, ya que nos permite conocer numerosos aspectos de los sistemas socioculturales humanos, como los económicos, tecnológicos, simbólicos, etc. (García Añino 2018; Hajduk y Lezcano 2005; Marchionni 2013; Mengoni Goñalons, 1999; Miotti, 1998 [1989], entre otros). El análisis de las estrategias de subsistencia y utilización de recursos por parte de los cazadores es uno de los temas centrales dentro de las investigaciones zooarqueológicas de Patagonia. En numerosos sitios arqueológicos tempranos de la Patagonia argentina y chilena (Figura 1) se han recuperado especímenes correspondientes a diferentes especies de camélidos (Marchionni 2013; Massone 2004; Miotti y Salemme 1999; Paunero et al. 2015, 2017, entre otros). La especie numéricamente dominante es el guanaco (Lama guanicoe), que fue el principal recurso económico desde el Pleistoceno final en adelante (Borrero 1990; Marchionni 2013; Martínez et al. 2016; Mengoni Goñalons 1999; Miotti 1998 [1989]; Miotti y Salemme 1999; Moscardi et al. 2020; Paunero et al., 2017; Rindel 2009, entre otros). No obstante, también se destaca el uso complementario de otros animales, entre los que se incluyen los caballos y

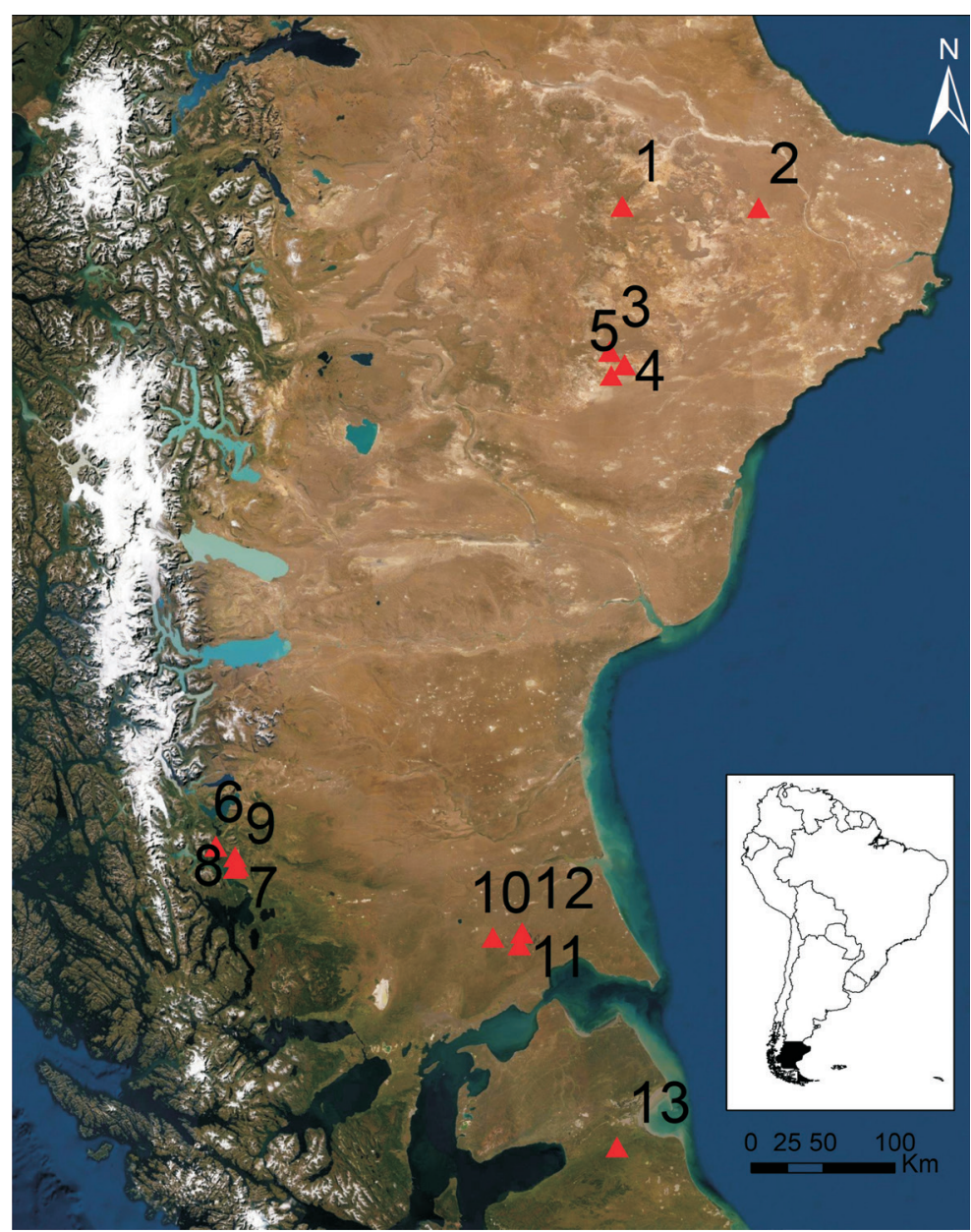

Figura 1. Ubicación geográfica de los sitios arqueológicos y paleontológicos considerados: 1. Los Toldos 1 y 2; 2 . Alero El Puesto 1; 3. Cueva Túnel; 4. El Ceibo 7; 5 . Casa del Minero 1; 6. Alero Dos Herraduras; 7. Cueva del Milodón; 8. Cueva Lago Sofía 1 y 4; 9 . Cueva del Medio; 10. Cerro Sota; 11. Cueva de Los Chingues; 12. Cueva del Puma y 13. Tres Arroyos 1.

Geographical location of the archaeological and paleontological sites considered: 1. Los Toldos 1 and 2; 2. Alero El Puesto 1; 3. Cueva Túnel; 4. El Ceibo 7; 5. Casa del Minero 1; 6. Alero Dos Herraduras; 7. Cueva del Milodón; 8. Cueva Lago Sofía 1 and 4; 9. Cueva del Medio; 10. Cerro Sota; 11. Cueva de Los Chingues; 12. Cueva del Puma, and 13. Tres Arroyos 1. 
otras especies de camélidos (Miotti y Salemme 1999, 2004; Moscardi et al. 2020).

En este sentido, otras especies extintas han sido identificadas en contextos arqueológicos, pertenecientes a la tribu Lamini, tales como Lama gracilis y Hemiauchenia paradoxa. Además de Cueva Túnel, se han detectado restos de Lama gracilis con evidencias de procesamiento en la Cueva 3 de los Toldos (nivel 11, Miotti 1998 [1989]), Piedra Museo (Unidad 6, Marchionni 2013; Miotti y Salemne 1999), y Casa del Minero 1 (Unidad 4, Paunero et al. 2017; Valiza Davis 2020), y sin evidencias de procesamiento en la Cueva 2 de Los Toldos (Mengoni Goñalons 1981), en las Unidades 4/5 de Piedra Museo (Marchionni 2013), Cueva 7 de El Ceibo (capa 12, Menegaz et al. 1989; Miotti 1998 [1989]) y la Unidad 3 inferior de Casa del Minero 1 (Paunero et al. 2017; Valiza Davis 2020). También se han recuperado restos de esta especie sin evidencias de procesamiento en Tierra del Fuego, en el sitio Tres Arroyos 1 (Labarca y Prieto 2009; Mengoni Goñalons 1987), y en Última Esperanza en los sitios Cueva Lago Sofía 1 y 4 y Cueva del Milodón (Labarca y Prieto 2009; Prieto y Canto 1997; Prieto y Labarca 2011) tratándose de depositaciones naturales. En el caso de H. paradoxa, se han recuperado restos con marcas antrópicas en el sitio Casa del Minero 1 (Paunero et al. 2017; Valiza Davis 2020). En Última Esperanza, Nami y Menegaz (1991), sobre la base del tamaño extremo de algunos restos dentro del género Lama, determinaron una especie Lama morfotipo $L$. oweni, que puede ser asignado a $H$. paradoxa. Estos restos muestran evidencias de procesamiento. Asimismo, estos autores mencionan que restos de un tamaño comparable se han recuperado en Tres Arroyos 1 (Nami y Menegaz 1991). Fuera de la región, se registran restos asignados a Hemiauchenia sp. en Paso Otero 5 (Martínez y Gutiérrez 2011) con posibles evidencias de procesamiento humano y a H. paradoxa en Pilauco (Labarca 2020), mientras que material similar referido a Paleolama está representado en Arroyo Seco 2 (Fidalgo et al. 1986) y Monte Verde (Casamiquela y Dillehay 1989). Como surge de esta discusión, existe considerable grado de debate acerca del estatus taxonómico de estas dos últimas especies (Gasparini et al. 2017; Labarca y Prieto 2009; Scherer et al. 2007; Weinstock et al. 2009). No obstante, distintos estudios de carácter morfológico han permitido discriminarlas a nivel específico (Labarca y Prieto 2009; Menegaz et al. 1989; Scherer et al. 2007). La característica distintiva entre los diferentes taxa es el tamaño de los especímenes, siendo uno de mayor (H. paradoxa) y otro de menor (L. gracilis) tamaño que el guanaco actual (Valiza Davis y Gasco 2019). Más allá del debate acerca de la clasificación sistemática, es importante conocer el tipo de explotación y la contribución relativa de estas especies a la subsistencia de los grupos humanos que colonizaron Patagonia. Así, el objetivo principal de este trabajo es presentar los resultados del análisis del conjunto óseo correspondiente a camélidos del componente inferior del sitio Cueva Túnel (CT), ubicado en la localidad arqueológica La María, provincia de Santa Cruz (Figura 1).

Las evidencias recuperadas en CT son relevantes porque reúnen algunos aspectos importantes para una discusión del proceso de poblamiento temprano de Patagonia meridional y de las estrategias de subsistencia implementadas por estos grupos. En primer lugar, el conjunto faunístico correspondiente a camélidos es abundante y se halla en buen estado de preservación. En segundo lugar, como veremos, este conjunto tiene evidencias inequívocas de procesamiento por parte de los humanos, por lo que permite realizar inferencias confiables acerca de la subsistencia humana en los comienzos del proceso de poblamiento del sur de Patagonia. En tercer lugar, la muestra de camélidos analizada comprende especímenes correspondientes a $L$. guanicoe, $L$. gracilis y $H$. paradoxa, lo que nos permitió discutir las semejanzas y diferencias en el tratamiento de estas especies por parte de los grupos humanos. Finalmente, la cronología del componente inferior ubica la muestra analizada en momentos inmediatamente previos a dos importantes cambios en los ecosistemas finipleistocénicos del sur de Patagonia: la extinción de varios de los taxones que conformaron la denominada "fauna Pleistocénica" (Borrero y Franco 1997) y el reemplazo de comunidades vegetacionales dominadas previamente por el estrato herbáceo por especies arbustivas hacia los 10200 años AP (De Porras 2010). Estos datos nos permiten discutir los patrones de subsistencia desarrollados por los primeros grupos humanos que poblaron los sectores interiores de Patagonia meridional en el contexto de un ambiente ecológico transicional. Para ello primero haremos una breve referencia a las características del sitio, para luego investigar las características tafonómicas de la muestra bajo estudio. Luego se presentarán los datos acerca de la representación de partes esqueletarias y de los patrones de procesamiento antrópico, integrando estos resultados en una discusión en la que se explorarán las principales tendencias en el uso de camélidos en el sitio. 


\section{Información General del Sitio y Cronología}

El sitio Cueva Túnel (CT) (48²4’S y 6852’W, $225 \mathrm{msm}$ ) se encuentra en la provincia de Santa Cruz, Patagonia argentina (Figura 1). Ubicado a la entrada de La María Quebrada, cercano al bajo colector de aguas de invierno-primavera. Se trata de una cueva con dos vestíbulos tapados por sedimentos, que convergen en un umbral. Presenta un ancho máximo de $6 \mathrm{~m}$ y una longitud máxima de 10,10 m desde la línea de goteo. Se ha excavado en el umbral mencionado y parte de los vestíbulos, registrando materiales en estratigrafía e identificando un componente inferior que presenta restos culturales asociados a la fauna. $\mathrm{Al}$ momento de la excavación inicial, la cueva se encontraba prácticamente saturada por sedimentos. Las excavaciones realizadas entre 2003 y 2010 se efectuaron en el umbral mencionado y parte de los vestíbulos, registrando materiales en estratigrafía e identificando la presencia de múltiples ocupaciones (Paunero 2009).

El sitio presenta una superficie total de $60,6 \mathrm{~m}^{2}$, con una potencia total de $260 \mathrm{~cm}$, aunque solo hay material cultural hasta los $120 \mathrm{~cm}$, siendo la superficie excavada total de $17,24 \mathrm{~m}^{2}$. El componente inferior analizado en este trabajo, conformado por las capas 8,9 y 10 , tiene una potencia que supera $\operatorname{los} 40 \mathrm{~cm}$ en algunos sectores y presenta fechados entre 10500 y 10400 años ${ }^{14} \mathrm{C} \mathrm{AP}$ (Tabla 1). Las fechas, obtenidas a partir de materiales faunísticos, son muy cercanas entre sí. Ello indica que la conformación del conjunto obedeció a eventos de sedimentación rápida ocurridos a finales de Pleistoceno (Paunero 2009). La matriz sedimentaria es ocre clara, con una granulometría areno-limosa con clastos. El origen de los sedimentos es principalmente eólico, espélico y antrópico. Debajo del componente inferior (unidades 8, 9 y 10), identificamos la Unidad 11, capa areno-limosa muy clara, que es estéril. Por encima del componente inferior registramos la Unidad 7 solamente en la cuadrícula A1. Se trata de un lente de sedimento areno-limoso compacto de 2 a $5 \mathrm{~cm}$ de espesor. La Unidad 6 es de un sedimento ocre de granulometría areno-limosa semejante a la subyacente, pero con menor cantidad de clastos. Estas unidades 6 y 7 representan una discontinuidad ocupacional con respecto al componente inferior (Paunero 2009).

El análisis de otras líneas de evidencia ofrece un contexto relevante para la discusión de la información faunística. El conjunto lítico está conformado por escasos restos, y cuenta con una alta proporción de instrumentos (Skarbun et al. 2015). Las evidencias de producción identificadas refieren a las etapas finales de manufactura lítica, no habiéndose hallado núcleos. Los instrumentos habrían ingresado ya formatizados. La materia prima más frecuente entre los artefactos formatizados es el ópalo translúcido. Su procedencia podría ser local (distancia menor a $15 \mathrm{~km}$ ), sin embargo, hasta el momento no se han identificado fuentes de este material en los alrededores de CT. En consecuencia, ello podría vincularse con las estrategias de movilidad y de producción artefactual en función de las actividades a realizar en el sitio, indicando una planificación de las tareas productivas con anticipación al consumo (Skarbun et al. 2015). Entre los artefactos formatizados se incluyen dos cuchillos, un cuchillo/raedera, una lasca retocada, una lámina retocada, una raedera, un chopping-tool/ percutor y numerosos productos de talla (Skarbun et al. 2015). Las evidencias provistas por el análisis funcional efectuado sobre los cuchillos, el cuchillo/ raedera y el chopping tool indican que estos fueron empleados en acciones de corte/percusión de carne, entre otros tejidos blandos, y en algunos casos muestran signos de haber estado en contacto con material óseo (Cueto 2015).

Por su parte, la evidencia arqueobotánica en el componente inferior incluye espículas de carbones

Tabla 1. Cronología del componente inferior de Cueva Túnel. Chronology of the lower component of Cueva Túnel.

\begin{tabular}{cccccc}
\hline Código & Fecha AP & $\begin{array}{c}\text { Mediana } \\
\text { cailbrada }\end{array}$ & Material Datado & Proveniencia & Referencia \\
\hline AA71148 & $10400 \pm 100$ & 12199 & $\begin{array}{c}\text { Segunda falange de Hippidion } \\
\text { saldiasi (AMS) }\end{array}$ & Unidad 10 & Paunero 2009 \\
\hline AA71147 & $10408 \pm 59$ & 12218 & $\begin{array}{c}\text { Fragmento hueso camélido } \\
\text { (AMS) }\end{array}$ & Unidad 10 & Paunero 2009 \\
\hline LP-1965 & $10420 \pm 180$ & 12181 & $\begin{array}{c}\text { Fragmento hueso Lama sp. } \\
\text { (LSC) }\end{array}$ & Unidad 8 & Paunero 2009 \\
\hline AA82496 & $10510 \pm 100$ & 12339 & $\begin{array}{c}\text { Fragmento hueso camélido } \\
\text { (AMS) }\end{array}$ & Unidad 8 & Paunero 2009 \\
\hline
\end{tabular}


dispersos, estando ausentes las estructuras de combustión (Frank 2011). También se identificaron pequeños fragmentos de madera correspondientes a los géneros Lycium y Berberis (Cueto y Andreoni 2016). Los estudios palinológicos han podido identificar polen de Poaceae, de Asteraceae subfamilia Asteroideae, de Ephedra frustillata y de Nassauvia, lo que estaría indicando una comunidad subarbustiva-graminosa que no tiene análogos en la actualidad (De Porras 2010).

Las características del conjunto lítico, junto con las primeras observaciones realizadas sobre los conjuntos óseos, han permitido proponer como hipótesis que el sitio habría funcionado como un espacio destinado al procesamiento primario de presas cazadas en las cercanías (Paunero et al. 2015).

\section{Materiales y Métodos}

Con el fin de evaluar la utilización humana de los camélidos presentes en el componente inferior de CT se utilizaron las siguientes variables: abundancia relativa de las especies de camélidos, frecuencia de partes esqueletarias, incidencia de factores naturales en la formación del conjunto y presencia de evidencias de procesamiento.

Para el relevamiento de estas variables se utilizó la metodología propuesta por Mengoni Goñalons (1999). Se procedió a la identificación taxonómica y anatómica de los elementos óseos. La determinación taxonómica fue efectuada sobre la base de comparaciones con colecciones de referencia de material arqueológico y paleontológico y análisis osteométricos multivariados. En primer lugar, se realizó un análisis osteométrico multivariado sobre primeras falanges de camélidos recuperados en el sitio usando Análisis de Conglomerados y Análisis de Componentes Principales (Valiza Davis y Gasco 2019), lo que permitió corroborar la presencia de tres especies de camélidos en el sitio. Es de destacar que algunas de estas asignaciones fueron confirmadas por análisis moleculares de dos especímenes de Lama gracilis del sitio Casa del Minero 1 (Metcalf et al. 2016). En segundo lugar, se efectuó un relevamiento comparativo del material postcraneal con material paleontológico depositado en la División Paleontología del Museo de La Plata (Valiza Davis 2020). También se comparó el material de Cueva Túnel con especímenes recuperados de camélidos extintos de sitio Casa del Minero 1 (Valiza Davis 2020). Asimismo, se utilizó material comparativo de Vicugna vicugna del Instituto Nacional de Antropología y Pensamiento
Latinoamericano (Valiza Davis 2020), dadas las similitudes osteológicas entre Lama gracilis y vicuña (Labarca y Prieto 2009). Finalmente, cabe aclarar que la asignación taxonómica del material se realizó con un criterio conservador. Aquellos materiales que no tenían caracteres diagnósticos o en los que estos eran dudosos fueron incluidos en la categoría Mamífero Grande, conformada en su mayoría por especímenes de Lama sp. No obstante, esta categoría no fue utilizada debido a la presencia de otras especies de megafauna como Hippidion saldiasi, Megatherium americanum, familia Mylodontinae y Arctotherium tarijensis.

En relación con la identificación anatómica, para cada elemento del esqueleto se consideraron las zonas diagnósticas definidas por Mengoni Goñalons (1999), así como la porción, lateralidad y el estado de fusión del hueso representado (Kaufmann 2009).

Luego se procedió a la evaluación de los agentes formadores de los conjuntos. Para ello se utilizó una perspectiva tafonómica (sensu Lyman 1994) en la cual se buscaron indicios de la operatoria de distintos agentes, tales como roedores, carnívoros, acción de raíces y pisoteo, entre otros (Fisher 1995). Se registraron los estadios de meteorización (Behrensmeyer 1978), así como la correlación entre la densidad mineral ósea (Elkin 1995) y la representación de partes esqueletarias.

Posteriormente, se estudiaron las evidencias de procesamiento, en la forma de marcas de corte y percusión, así como la frecuencia de huesos termoalterados, con el fin de evaluar los tipos y la intensidad de las actividades desarrolladas por los humanos en referencia al conjunto. Asimismo, se consignaron los tipos de marcas de corte sobre la base de su localización topográfica en las superficies de los huesos, su orientación primaria/profundidad/largo, orientación secundaria y frecuencia (sensu Mengoni Goñalons 1999), realizándose dibujos mudos de cada daño registrado. Estas marcas fueron comparadas posteriormente con daños de etiología conocida (Binford 1981) para determinar las actividades responsables de las mismas: remoción de cuero, desarticulación y descarne. Como ya se mencionó, este tipo de análisis permite discutir con mayor grado de detalle la variabilidad presente en una muestra determinada y se constituye en un buen indicador de las etapas de procesamiento más representadas (Rindel 2009). Por lo tanto, es una línea de evidencia que potencialmente puede contribuir en mayor medida que otras a explorar aspectos relacionados con el rango de actividades vinculadas con el aprovechamiento de la fauna. Se registró la presencia de termoalteraciones de acuerdo 
a criterios macroscópicos, como el color que presenten los huesos y las alteraciones de sus superficies (Mengoni Goñalons 1999). Los resultados se presentan en términos de medidas de abundancia taxonómica (MNI y NISP) y medidas de abundancia de partes (MNE, MAU y \% de MAU) (Binford 1981; Grayson 1984; Lyman 1994; Mengoni Goñalons 1999, entre otros).

Por último, existen índices que nos ayudan a interpretar la toma de decisiones de los grupos del pasado con respecto a la selección y transporte de partes esqueletarias. Estos son marcos de referencia que permiten comparar los huesos hallados en el registro con valores derivados del rendimiento económico de los animales que conformaron la base de recursos. Particularmente relevantes en este trabajo son el Índice de Carne -MGUI- (Borrero 1990) y el Índice de Médula -IM- (Mengoni Goñalons 1996), ambos elaborados a partir de huesos de guanaco. También se utilizaron el Índice de Médula Ínsaturada -IMNS- (Morin 2007), obtenido a partir del cociente del volumen de la cavidad medular y la proporción de ácidos grasos insaturados de los huesos de las extremidades del caribú, y el Índice de Secado -IS- (De Nigris y Mengoni Goñalons 2004). Con el objetivo de comparar las frecuencias de partes esqueletarias con los diferentes índices discutidos previamente, se utilizó el coeficiente de correlación de Spearman (rho de Spearman), que usa una escala ordinal. Los diferentes marcos de referencia fueron correlacionados con el MAU porcentual obtenido para cada taxón.

\section{Resultados}

\section{Representación taxonómica y anatómica}

Como fuera señalado, este trabajo se centra específicamente en los camélidos (Tabla 2). Entre estos se encuentra el guanaco (Lama guanicoe), que corresponde a la especie dominante de acuerdo al Número Mínimo de Especímenes (NISP=541) y se destaca como único representante viviente en la actualidad. En segundo lugar en términos de NISP se destaca en taxón extinto $L$. gracilis (NISP=49), seguido por Hemiauchenia paradoxa (NISP=18). Un elevado número de elementos (NISP=1096) no presentaban atributos diagnósticos suficientes para ser asignados a una especie en particular y fueron incluidos en la categoría Mamífero Grande, en su mayoría conformada por restos de camélidos. Además, el conjunto del componente inferior de $\mathrm{CT}$ presenta una elevada cantidad de taxones. En este sentido es importante destacar la presencia de otras especies de fauna extinta, tales como Hippidion saldiasi, Megatherium americanum y familia Mylodontinae, aunque corresponden a un reducido porcentaje de la muestra total. Asimismo, también se registró la presencia de carnívoros, tanto extintos como actuales. Entre los primeros se encuentra Panthera onça mesembrina y Arctotherium sp., mientras que en el segundo caso se registró la presencia de puma y zorro. Como veremos en el acápite de la información tafonómica, la presencia de estos carnívoros nos alertó acerca de la necesidad de evaluar cuidadosamente los tipos de daño presentes en el conjunto, con el fin de determinar los agentes responsables de la génesis de los depósitos. En particular el registro del jaguar extinto, capaz de generar un elevado grado de destrucción ósea y que además ocupaba en alternancia los mismos espacios que los grupos humanos (Martin 2013), podría estar indicando el uso de CT como madriguera en algún momento de su historia ocupacional.

En relación con la representación de partes esqueletarias, en el caso del guanaco (Tabla 2) se observó una alta frecuencia ( $>75 \%$ del MAU) de radioulna y metatarso proximal y diáfisis de huesos largos (húmero y tibia). Con una representación intermedia se encuentran el atlas, epífisis proximal de fémur, epífisis distal de metapodios, rótulas y calcáneos. El resto de los elementos, entre los que se incluye restos del esqueleto axial y falanges, se encuentra por debajo del $50 \%$ del MAU. A partir de distintas regiones de húmero derecho se pudo determinar un Número Mínimo de Individuos (MNI) de cuatro guanacos. Al menos uno de ellos corresponde a una cría, dado que se recuperó una epífisis distal de húmero no fusionada, que pertenece al grupo de fusión temprana (sensu Kaufmann 2009). Considerando la relación NISP/MNE para el conjunto de guanacos, se observa un alto grado de fragmentación (3,09). Esto podría estar indicando a priori un importante procesamiento antrópico de este taxón, o una fuerte actividad tafonómica (p.ej., meteorización).

La representación de partes esqueletarias de L. gracilis (Tabla 2) es más acotada cuando se la compara con la del guanaco. De esta forma, se registra una alta frecuencia de carpianos, tarsianos, rótula $\mathrm{y}$ falanges primeras (100\% del MAU), junto con representación media (50\% del MAU) de partes de cráneo, pelvis, costillas, calcáneo, astrágalo, falanges segunda y tercera, y huesos largos. Por último, se ha observado una baja frecuencia ( $<20 \%$ del MAU) de elementos correspondientes a vértebras cervicales, metapodios y sesamoideos. El MNI correspondiente a Lama gracilis es de dos individuos, determinados 
Tabla 2. Representación anatómica de los camélidos de Cueva Túnel.

Frequency of skeletal parts of Cueva Túnel camelids.

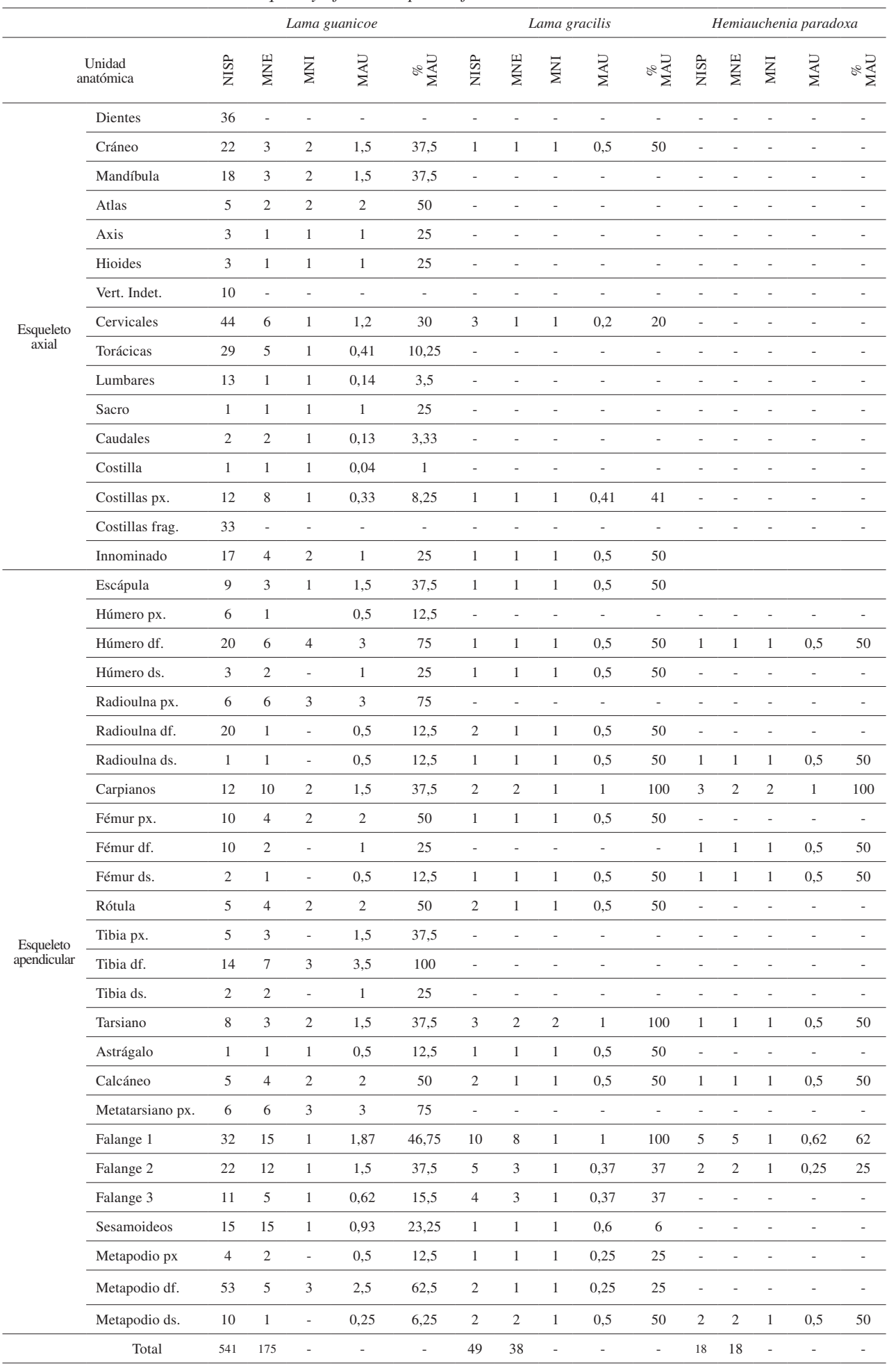


a partir de la presencia de dos cuboides izquierdos. De acuerdo a la relación NISP/MNE para $L$. gracilis se observa una fragmentación moderadamente baja $(=1,28)$.

Por último, en el caso de Hemiauchenia paradoxa (Tabla 2) se destaca su reducida representación de partes esqueletarias, limitada solamente a algunos elementos del esqueleto apendicular. De esta forma, se registró una alta frecuencia de carpianos, seguido de primeras falanges. También hay una representación media de elementos diafisiarios de húmero y radioulna, así como segmentos distales de metapodio y tarsianos. Para este taxa, la presencia de dos unciformes izquierdos permitió determinar un MNI de dos individuos. La relación NISP/MNE para $H$. paradoxa es baja $(=1)$.

En resumen, se observa una representación equilibrada de partes de guanaco, el segundo taxón en importancia es $L$. gracilis y en tercer lugar hay una baja frecuencia de partes correspondiente a H. paradoxa. Ello sería consistente con el ingreso de animales relativamente completos al sitio en el caso de las dos primeras especies, y posiblemente implicando el transporte de segmentos seleccionados de la carcasa en el caso de H. paradoxa.

\section{Análisis tafonómico}

La información tafonómica indica que, en general, la muestra se encuentra poco afectada por agentes y procesos naturales. En el caso de la meteorización, tal como se observa en la Figura 2, más del $90 \%$ de los elementos no sobrepasa el estadio 2, independientemente del taxón considerado. Asimismo, tanto para guanaco como para $L$. gracilis, la mayor parte de los huesos se concentra en el estadio $0 \mathrm{y}$ la representación en cada categoría subsiguiente va descendiendo. En el caso de H. paradoxa, el estadio más representado es el 1. Estos datos permiten afirmar que este proceso no ha sido relevante en la formación y modificación del conjunto, posiblemente relacionado con la protección conferida por el entorno de cueva del sitio. En relación con el patrón de meteorización, se utilizó la densidad mineral ósea como herramienta para determinar en qué medida los conjuntos fueron afectados por procesos de destrucción ósea, utilizándose para ello los valores publicados en el trabajo de Elkin (1995). Los análisis realizados muestran que la densidad mineral ósea no explica el patrón de partes esqueletarias presentes, ya que, para el caso del guanaco, la correlación de Spearman entre el MAU porcentual y DMO es baja y no significativa $(r=0,20$; $\mathrm{p}=0,26$ ). Lo mismo ocurre para el caso de L. gracilis $(\mathrm{r}=0,36 ; \mathrm{p}=0,14)$ y Hemiauchenia $(\mathrm{r}=0,28 ; \mathrm{p}=0,50)$, cuyas correlaciones también resultan no significativas.

Como fuera mencionado en la sección de estructura taxonómica del conjunto, se registraron varios taxa correspondientes a carnívoro. De esta forma, uno de los elementos a considerar fue de qué manera estas

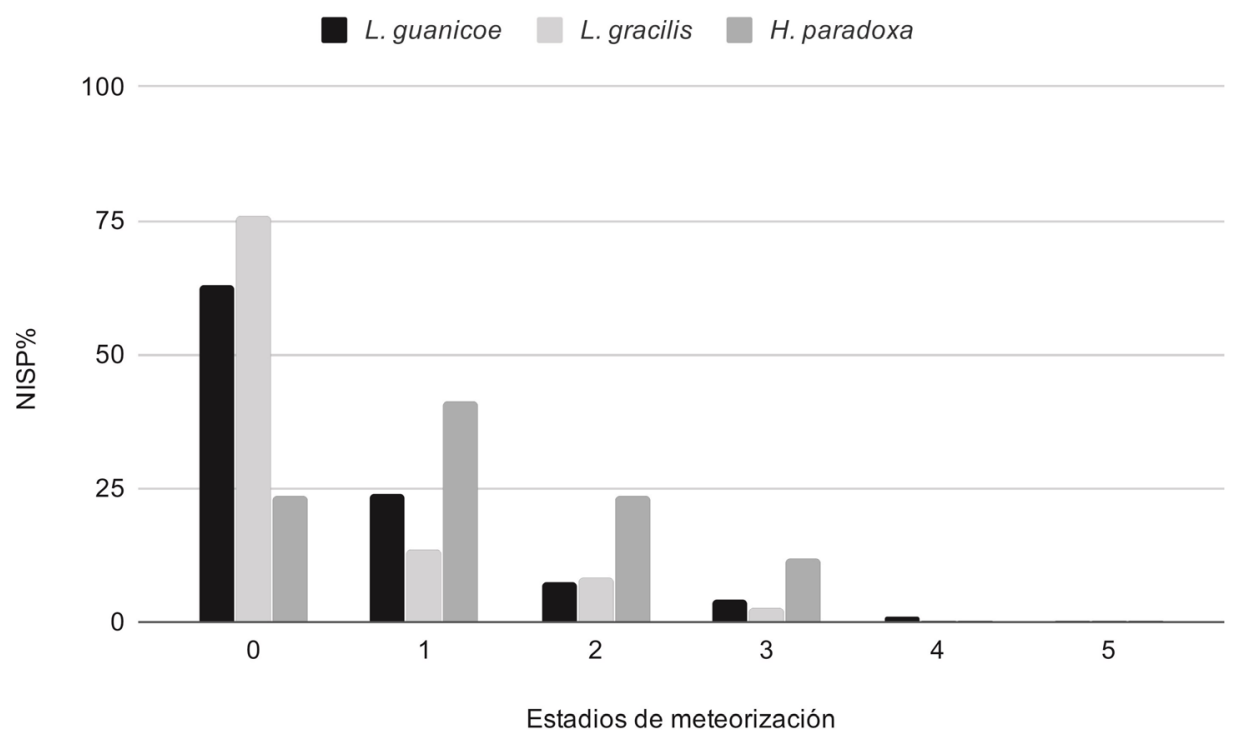

Figura 2. Estadios de meteorización de los restos de camélidos de Cueva Túnel (\%NISP).

Weathering stages in camelid remains from Cueva Túnel (\% NISP). 
especies influyeron en la conformación del conjunto. En este sentido, en CT se observa que este tipo de daño es considerablemente bajo (Tabla 3). Las huellas, reconocidas sobre epífisis y diáfisis de huesos largos, se ubican mayoritariamente en la categoría de pozos (pits) y hoyuelos de pequeñas dimensiones (punctures), aunque también se registran surcos producidos por el arrastre de los dientes (scoring) y evidencias de digestión. Se sugiere, a partir de lo analizado, que los agentes involucrados fueron carnívoros de pequeño porte, tales como zorros. Asimismo, el escaso daño registrado aquí queda evidenciado en la Figura 3, donde se presentan datos de frecuencias de daño por carnívoro sobre diferentes especies en una serie de sitios de Patagonia austral, en los que se ha inferido que el agente responsable es la pantera patagónica (Martin 2008). En general el porcentaje se encuentra entre el 10 y el $20 \%$, y llega hasta el $50 \%$ en el caso de conjuntos puramente paleontológicos interpretados como paleo-madrigueras de este felino, como en el caso de Cueva de los Chingues (Martin 2008). No obstante, en el caso de CT solo el 1,12\% de los huesos asignados a camélido presenta huellas de carnívoro (n=7, Figura 3). De esta forma, la línea
Tabla 3. Frecuencia de agentes y procesos tafonómicos en camélidos de Cueva Túnel (\% NISP).

Frequency of agents and taphonomic processes in Cueva Túnel camelids (\%NISP).

\begin{tabular}{lccc}
\hline & $\begin{array}{c}\text { Lama } \\
\text { guanicoe }\end{array}$ & $\begin{array}{c}\text { Lama } \\
\text { gracilis }\end{array}$ & $\begin{array}{c}\text { Hemiauchenia } \\
\text { paradoxa }\end{array}$ \\
\hline Carnívoro & 1,11 & 4 & - \\
\hline Roedor & 3,14 & 4 & - \\
\hline $\begin{array}{l}\text { Marcas de } \\
\text { raíces }\end{array}$ & 2,35 & - & 5,55 \\
\hline Manganeso & 34,25 & 14 & 44,44 \\
\hline Adherencias & 21,66 & 22 & 16,66 \\
\hline Teñido & 0,37 & - & - \\
\hline $\begin{array}{l}\text { Periostio } \\
\text { Espícula de } \\
\text { carbón }\end{array}$ & 0,74 & - & - \\
\hline
\end{tabular}

de evidencia más fuerte para evaluar el aporte de los carnívoros a la conformación y modificación del conjunto -las evidencias de dientes sobre las superficies de los huesos- indica que este no fue importante. No obstante, no es posible descartar totalmente la actividad potencial de estos agentes acumuladores, dado que es evidente que al menos

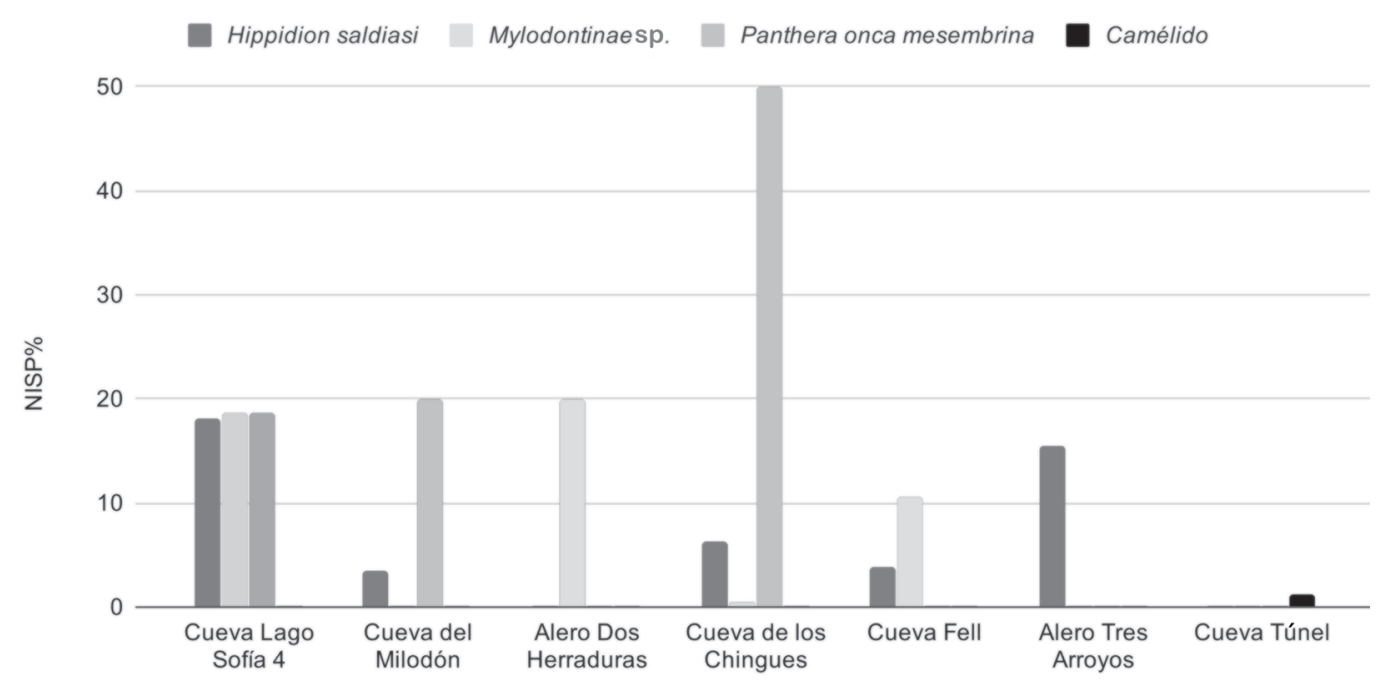

Figura 3. Comparación entre el porcentaje de elementos de megafauna con huellas de carnívoro en diferentes conjuntos correspondientes a la transición Pleistoceno-Holoceno de Patagonia y el componente inferior de Cueva Túnel. Datos de los sitios Cueva Lago Sofía 4, Cueva del Milodón, Alero Dos Herraduras, Cueva de los Chingues, Cueva Fell y Alero Tres Arroyos. Reformulado de Martin (2008).

Percentual comparison of different megafaunal species with carnivore marks in different Pleistocene-Holocene transition assemblages from Patagonia and those from the lower component of Cueva Túnel. Sites considered: Cueva Lago Sofía 4, Cueva del Milodón, Alero Dos Herraduras, Cueva de los Chingues, Cueva Fell and Alero Tres Arroyos. Reformulated from Martin (2008). 
carnívoros pequeños estuvieron implicados en la modificación de algunos especímenes.

Asimismo, otros tipos de marcas también están presentes en bajos porcentajes, como es el caso de roedores y raíces (Tabla 3). En mayores porcentajes se registraron frecuencias relativamente elevadas de manchas de óxido de manganeso en las tres especies, indicando la existencia de condiciones de humedad dentro de CT hacia el momento de conformación del componente inferior (Tabla 3). Finalmente, también se encuentran las adherencias, que corresponden a depósitos de carbonato de calcio, posiblemente provenientes de la disolución de la roca de caja, que blanquean la superficie de una elevada proporción de la muestra (Tabla 3). Es posible que la presencia de agua en el interior de la cueva facilitara la disolución de la roca de caja. Ambos tipos de modificaciones se observan en algunos huesos, lo que sugiere un mecanismo común. Además, solo para guanaco, se registraron otras alteraciones en las superficies óseas (Tabla 3) entre ellas se destacan aquellos elementos "teñidos". El origen de la coloración de estos elementos (fuego, manganeso u otro) es incierto. También se determinaron restos con espículas de carbón adheridas y otros con restos de periostio.

\section{Evidencias de procesamiento antrópico}

Los tres taxones muestran evidencias de procesamiento antrópico en frecuencias variables: guanaco $(14,65 \%, \mathrm{~N}=74)$, Lama gracilis $(20,40 \%$, $\mathrm{N}=10)$ y Hemiauchenia paradoxa $(5,55 \%, \mathrm{~N}=1)$. La distribución de los diferentes tipos de marcas de procesamiento, sin embargo, es diferente. De esta forma, en el guanaco predominan las marcas de corte, seguidas por las combinadas de corte y percusión y finalmente aquellos elementos que presentan solo percusión (Figuras 4 y 5A-B). En el caso de L. gracilis, por el contrario, se observa un predominio de evidencias de percusión, de corte, seguidas por corte y percusión (Figuras 4 y 5C). Finalmente, solo se detectaron evidencias de corte en $H$. paradoxa (Figura 4).

Al efectuar un análisis detallado de los tipos de marcas representadas de acuerdo a diferentes variables, lo que se puede observar en primer lugar es que la mayor parte de las evidencias de corte corresponde a la categoría de pocas ( $>5$ marcas por hueso) (Mengoni Goñalons 1999). Estas se encuentran mayoritariamente concentradas en determinados sectores de los huesos, en general próximos a articulaciones. Asimismo, revisando la localización y frecuencia de cada marca y, en algunos casos, comparándolas con la tipología producida por Binford (1981) a partir de evidencia etnoarqueológica, se pudo asignar a cada marca de corte una funcionalidad específica. En el caso del guanaco, encontramos una mayor diversidad de actividades, entre las que se destaca el descarne y desarticulación de presas, pero también evidencias

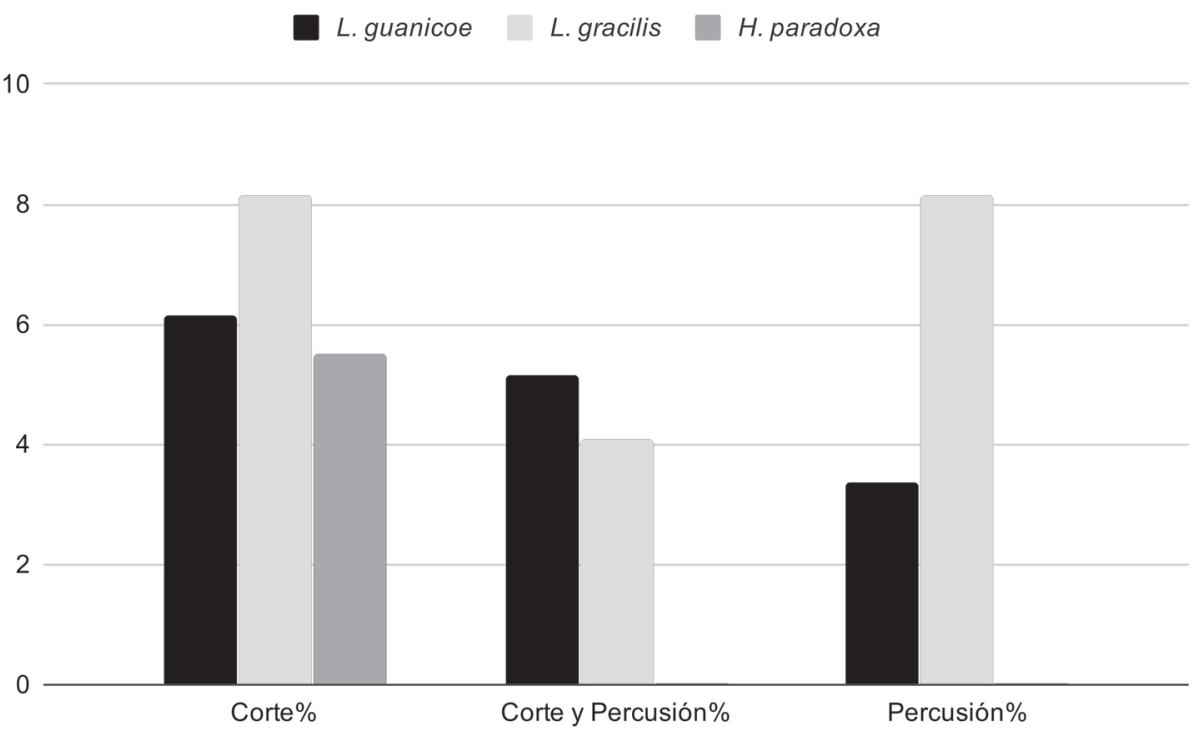

Figura 4. Frecuencia de evidencias de procesamiento sobre las tres especies de camélidos (\%NISP).

Frequency of processing marks on the three species of camelids (\%NISP). 
A.

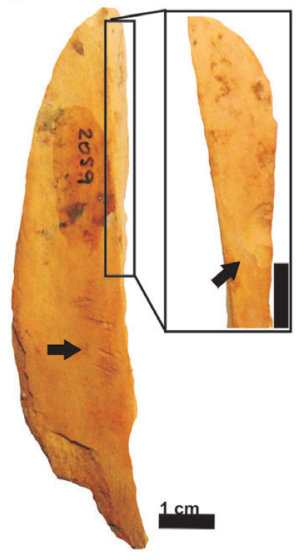

B.

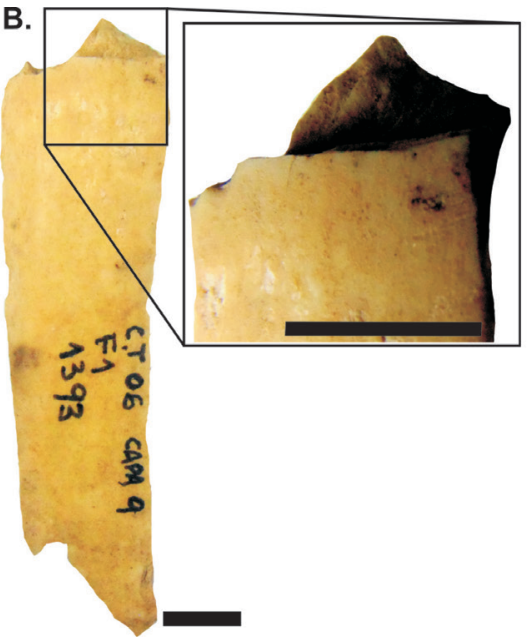

c.

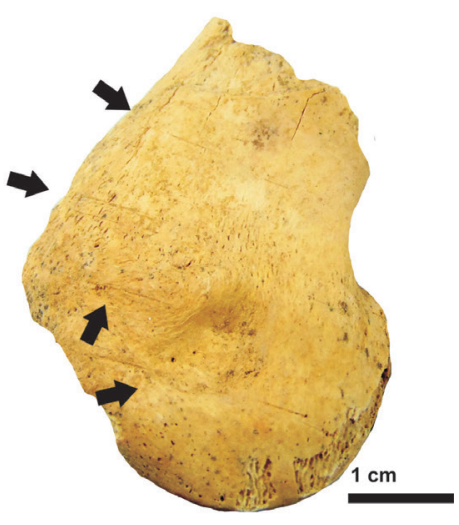

Figura 5. Huellas de procesamiento: (A) Diáfisis de radioulna de guanaco con marcas de corte y negativo de impacto; (B) Diáfisis de radioulna de guanaco con marcado perimetral; (C) Cóndilo lateral de húmero de Lama gracilis con numerosas marcas de corte.

Processing marks: (A) Guanaco radioulna shaft with cut marks and conchoidal flake scar; (B) Guanaco radioulna diaphysis with perimetral marking; (C) Lateral condyle of Lama gracilis humerus with multiple cut marks.

de segmentación de unidades esqueletarias mayores y de remoción de cuero (Figura 6). Para los otros dos taxa, la variedad de actividades es más restringida, puesto que la mayor parte de los cortes corresponde a actividades de desarticulación de los huesos (Figura 6). Finalmente, la Figura 7 representa los tipos de marcas que pudieron ser reconocidas en la muestra analizada a partir de su similitud con los tipos de daño tipificados por Binford (1981) a partir de su trabajo con los Nunamiut.

Respecto de las acciones de percusión, las mismas solo se evidenciaron en los elementos de guanaco y de L. gracilis. La presencia de negativos, estrías, hoyos de percusión y lascas adheridas así como evidencias de marcado perimetral dan cuenta de las fracturas intencionales llevadas a cabo en el sitio. La mayoría de estas fracturas indica actividades de extracción de médula. Asimismo, en algunos casos la suma de otros indicadores, como las evidencias de raspado y pulido, señala que los elementos óseos fueron trabajados con el objetivo de formatizar artefactos. En este sentido, es destacable la presencia de un fragmento con punta roma en su extremo, un punzón doble sobre hueso largo de guanaco, un punzón sobre metapodio de guanaco y un "machacador" confeccionado sobre un fémur de L. gracilis (Paunero et al. 2010; Valiza Davis 2020).

En el conjunto las evidencias de termoalteración son escasas: el 16,36\% de los huesos de guanaco
$(\mathrm{N}=88)$, el $24 \%$ de los huesos de Lama gracilis $(\mathrm{N}=12)$ y el $5,55 \%$ de los huesos de Hemiauchenia paradoxa $(\mathrm{N}=1)$ presentan evidencias de haber estado sometidos a la acción del fuego (Figura 8). Tanto los elementos asignados a guanaco como a $L$. gracilis muestran todos los estadios de este tipo de modificación, y en ambos taxones predominan los elementos carbonizados. En el caso de H. paradoxa solamente se registraron estadios iniciales de termoalteración, correspondientes a la categoría de quemado. Son llamativas las evidencias de huesos termoalterados dada la ausencia de estructuras de combustión en el componente inferior de CT (Frank 2011).

Con el objetivo de obtener datos acerca de la toma de decisiones detrás de la representación de partes esqueletarias observada en los distintos taxones, se efectuaron correlaciones entre diferentes índices y el MAU porcentual de cada especie (Tabla 4). Es importante señalar que estos resultados deben ser tomados con precaución, debido a que la muestra de partes esqueletarias de Lama gracilis y Hemiauchenia paradoxa está compuesta por pocos especímenes. Ello hizo que las correlaciones estuvieran basadas en poca cantidad de elementos: se tomaron 30 pares para L. guanicoe, 15 para L. gracilis y nueve para H. paradoxa. No obstante, se presenta el análisis de manera exploratoria y meramente con el objetivo de destacar tendencias generales en la selección de 


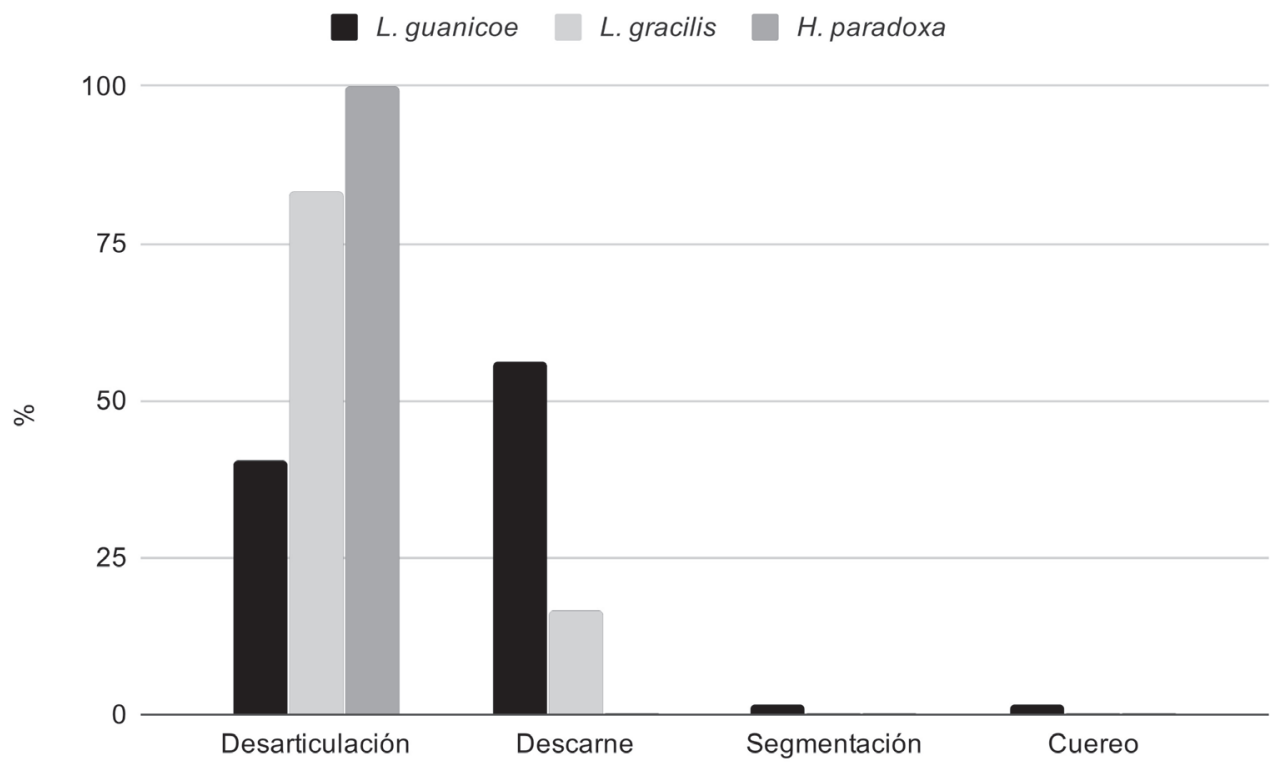

Figura 6. Frecuencia de los diferentes tipos de marcas de corte sobre elementos correspondientes a camélidos de acuerdo a la actividad realizada (\% NISP).

Frequency of the different cut marks types on elements corresponding to camelids according to the activity carried out $(\%$ NISP).

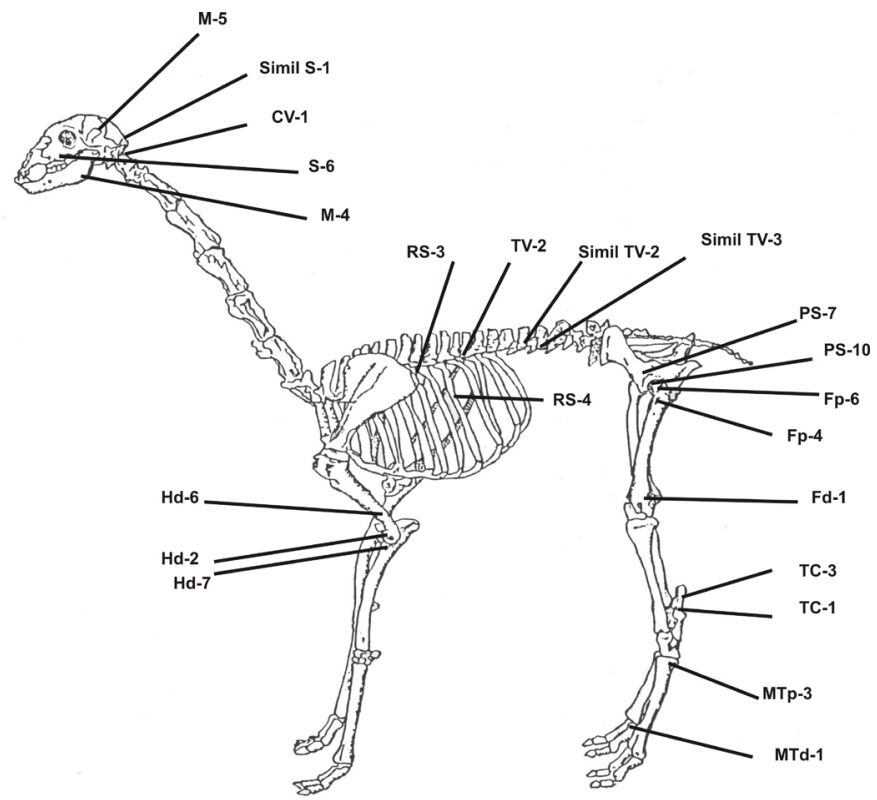

Figura 7. Tipos de marcas propuestas por Binford (1981) reconocidas en los restos de camélidos de Cueva Túnel.

Types of marks proposed by Binford (1981) and recognized in the camelid remains from Cueva Túnel. 


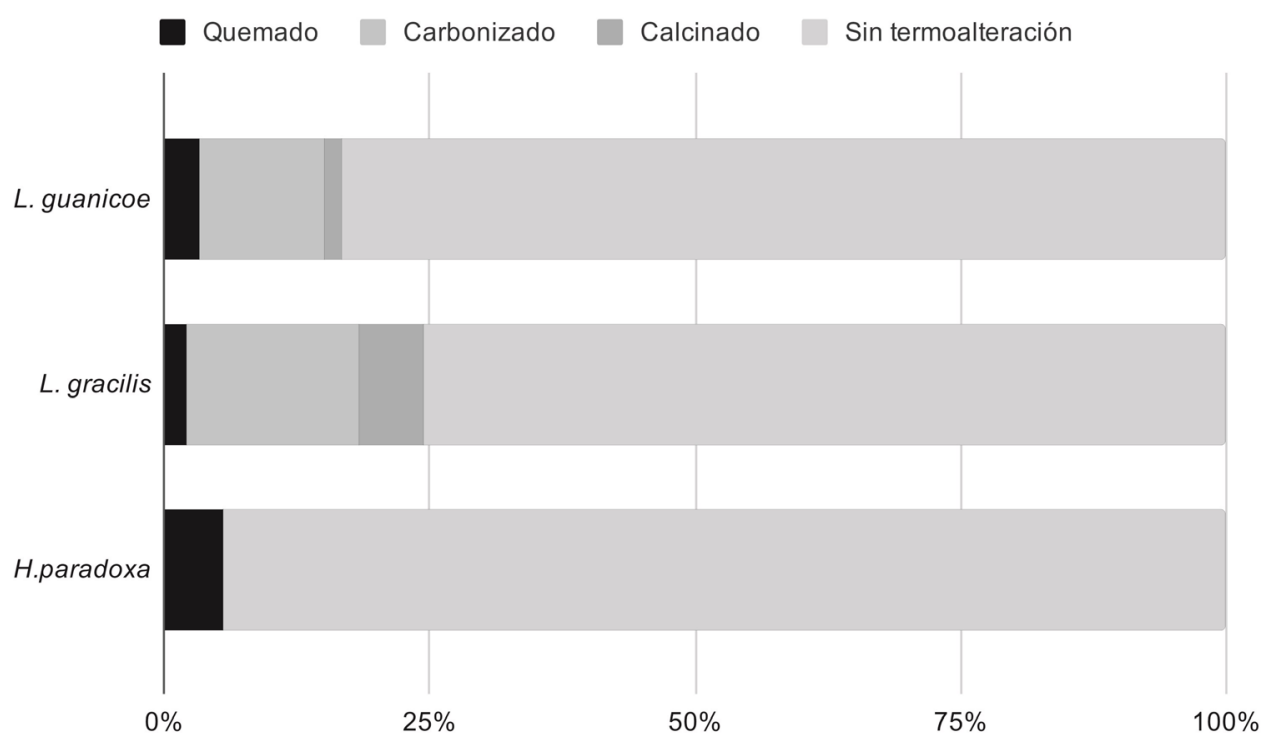

Figura 8. Frecuencia de los diferentes estadios de termoalteración sobre los huesos de las tres especies de camélidos (\% NISP).

Frequency of Thermoalteration stages on the bones of three camelid species (\% NISP).

las diferentes unidades anatómicas. De esta forma, se tuvieron en cuenta los datos tomados de Borrero (1990) para el Índice de Carne (MGUI) y el Índice de Médula (Mengoni Goñalons 1996), para el Índice de Médula No Saturada (IMNS) se siguió a Morin (2007) y para el Índice de Secado (IS) lo propuesto por De Nigris y Mengoni Goñalons (2004). Los resultados obtenidos, indican ausencia de correlación para casi todos índices. La única correlación significativa, alta y negativa se estableció entre guanaco e Índice de Secado (IS). Esto señala la ausencia de huesos aptos para secado de carne. Asimismo, en el caso del guanaco existe una correlación alta respecto del Índice de médula no saturada (IMNS), pero esta resulta no significativa. No obstante, el valor de $p$ se aproxima a 0,05 , por lo tanto, este resultado podría indicar cierta selección de elementos con un importante contenido de médula insaturada.

\section{Discusión}

El análisis presentado permitió confirmar un número de tendencias previamente observadas en el caso de otros conjuntos correspondientes a momentos de la transición Pleistoceno-Holoceno en Patagonia. En este sentido, se han recuperado restos de las tres especies de camélidos, algunos de ellos con evidencias de procesamiento, en diferentes sitios de la Meseta Central, Tierra del Fuego y Última Esperanza, tales como Los Toldos (cuevas 2 y 3), Piedra Museo (U6), Casa del Minero (U 4), Tres Arroyos 1, Cueva del Medio y fuera del área de estudio en Paso Otero 5, donde se identificó un fragmento de tibia de Hemiauchenia sp. con posibles fracturas antrópicas (Labarca y Prieto 2009; Marchionni 2013; Martínez y Gutiérrez 2011; Mengoni Goñalons 1981, 1987; Miotti 1998 [1989]; Nami y Menegaz 1991; Paunero et al. 2017; Valiza Davis 2020). A ello se suma la presencia de otra fauna extinta (fundamentalmente caballo y milodón) con evidencias de procesamiento, lo que apoya la idea de interacciones de tipo presa-predador entre humanos y megafauna (Marchionni 2013; Martin 2013; Miotti y Salemme 1999; Moscardi et al. 2020; Paunero et al. 2017; Valiza Davis 2020, entre otros). No obstante, entre los tres taxones de camélidos recuperados, la especie dominante en Cueva Túnel es el guanaco de acuerdo al Número Mínimo de Especímenes (NISP). Este hallazgo es consistente con los datos provenientes de otros sitios de la Meseta Central de Santa Cruz y otros sectores de la Patagonia meridional durante el proceso de colonización inicial, que indican una preponderancia de esta especie y la utilización 
Tabla 4. Coeficientes de correlación: MAU\% con el Índice de Carne (MGUI), el Índice de Médula (IM), el Índice de Médula No Saturada (IMNS) y el Índice de Secado (IS). Correlation coefficients: MAU\% with the Meat Utility Index (MGUI), the Marrow Index (IM), the Unsaturated Marrow Index (IMNS), and the Drying Utility Index (IS).

\begin{tabular}{|c|c|c|c|c|}
\hline & $\begin{array}{c}\% \\
\text { MAU/MGUI }\end{array}$ & $\begin{array}{c}\% \\
\text { MAU/IM }\end{array}$ & $\begin{array}{c}\% \\
\text { MAU/IMNS }\end{array}$ & $\begin{array}{c}\% \\
\text { MAU/IS }\end{array}$ \\
\hline Lama guanicoe & $\mathrm{r}=-0,29 ; \mathrm{p}=0,11$ & $\mathrm{r}=0,48 \mathrm{p}=0,29$ & $r=0,79 ; p=0,08$ & $\mathrm{r}=-0,85 ; \mathrm{p}=0,01$ \\
\hline L. gracilis & $\mathrm{r}=-0,18 ; \mathrm{p}=0,47$ & $\mathrm{r}=-0,70 \mathrm{p}=0,4$ & $r=0,04 ; p=0,94$ & $r=-0,67 ; p=0,3$ \\
\hline $\begin{array}{l}\text { Hemiauchenia } \\
\text { paradoxa }\end{array}$ & $r=-0,09 ; p=0,8$ & $\mathrm{r}=0,2 \mathrm{p}=0,65$ & $r=-0,07 ; p=0,88$ & - \\
\hline
\end{tabular}

complementaria de otros animales (Miotti 1998 [1989]; Miotti y Salemme 1999, 2004). El Número Mínimo de Individuos (MNI) apoya las tendencias obtenidas a partir del NISP, dado que se recuperaron restos correspondientes a cuatro guanacos, mientras que Lama gracilis y Hemiauchenia paradoxa se hallan representadas por dos individuos cada una. Asimismo, es importante resaltar que Cueva Túnel en particular y la Meseta Central en general presentan ciertas diferencias, en términos de la utilización de la fauna por parte de los primeros grupos humanos, con respecto a lo que se observa en otras partes de Patagonia. En los sitios de Última Esperanza y Palli Aike, por ejemplo, la explotación de la fauna extinta parece haber sido muy poco intensa, como lo demuestra la evaluación tafonómica de diferentes sitios (Cueva del Milodón, Cueva de los Chingues, Cueva del Puma, Cueva del Medio, Cerro Sota, Cueva Dos Herraduras, ver Bird 1988; Borrero 1999; Martin et al. 2011, entre otros).

Aunque otros taxones extintos también se encuentran presentes en el componente inferior de CT, (Paunero et al., 2015, 2017), los análisis presentados se limitaron al estudio de los camélidos, debido a la importancia que tuvieron desde los comienzos del poblamiento. De esta forma, el examen de las diferentes variables tafonómicas ha permitido señalar, en primer lugar, que la incorporación del material a la cueva es fundamentalmente de origen antrópico y no por carnívoros, a pesar de la presencia de jaguar y oso extinto, puma y zorros. En especial la presencia de una especie de jaguar extinto, Panthera onça mesembrina, potencialmente pudo tener un papel importante en la modificación del conjunto. Se ha observado que esta especie ocupaba con frecuencia cuevas, transportando y consumiendo presas en entornos relativamente protegidos, así como cubil para la crianza de cachorros (Martin 2008; Martinic 1996). Observaciones realizadas en jaguares modernos indican que los mismos poseen una dentición muy robusta y poderosa, la segunda después de las hienas (Del Moral Sachetti et al. 2011; Morales-Mejía et al. 2010; Schaller y Vasconcelos 1978). Esta característica le permite a esta especie formas de matar a las presas que son únicas entre los felinos, tales como la perforación del cráneo con los caninos (Schaller y Vasconcelos 1978), así como patrones de daño óseo muy intenso. El taxón extinto era de mayor tamaño que la especie actual (Prevosti y Martin 2013), sugiriendo un potencial de destrucción de huesos aún mayor. No obstante, la comparación de la frecuencia y tipo de daño de carnívoros del sitio con otros conjuntos, tanto arqueológicos como paleontológicos, como los analizados por Martin $(2008,2013)$, indican una muy baja frecuencia para CT y un tipo de daño muy ligero circunscrito a solamente a pequeños hoyuelos y punctures. Concordante con esto, tanto la baja frecuencia de elementos meteorizados, de huesos con marcas de raíces y con daño por roedores indican que el estado de la muestra es bueno, permitiendo la determinación de marcas de procesamiento. La ausencia de correlación del MAU\% de guanaco, L. gracilis y $H$. paradoxa con la densidad mineral indica que no ha habido procesos de destrucción diferencial que sean relevantes a la hora de explicar el patrón de partes esqueletarias presentes.

El guanaco y en menor medida $L$. gracilis muestran una distribución generalizada de partes esqueletarias, indicando que las carcasas de ambas especies ingresaron relativamente completas. Esto se manifiesta en el caso de este último taxón, por ejemplo, en la ausencia de correlación entre el MAU porcentual y los diferentes índices utilizados para intentar explicar su frecuencia de partes esqueletarias. En el caso del guanaco, con una muestra bastante más importante, se observó una correlación entre la frecuencia de partes esqueletarias y el Î́ndice de Secado y una correlación marginalmente significativa con el Índice de Médula No Saturada. 
Esto plantea la posibilidad de que la representación de elementos del guanaco esté vinculada en este componente con, por un lado, la ausencia de huesos aptos para secado, posiblemente debido a su transporte desde el sitio y por otro, con la selección de huesos de acuerdo a la calidad de la médula, con alto contenido de grasas no saturadas. En el caso de Hemiauchenia paradoxa, el patrón de representación de partes es muy restringido y no se detectaron correlaciones significativas. Es interesante notar que hay importantes diferencias de tamaño entre $H$. paradoxa $(300 \mathrm{~kg}$, Fariña et al. 2013), Lama guanicoe (100 kg) y L. gracilis (50 kg, Cajal et al. 2010). Es posible que en el caso de $H$. paradoxa, como en otros taxones de megafauna (sensu Martin 1967), estuvieran operando constreñimientos de transporte. En este sentido, la información actualística indica que los humanos normalmente transportan un rango máximo de pesos ubicado entre el 70-80 $\%$ de su peso corporal (Morin y Ready 2013). Para individuos con un peso estimado de entre 70 y $90 \mathrm{~kg}$ para mujeres y hombres, respectivamente, ello implica cargas máximas de $50-60 \mathrm{~kg}$. Esto es entre $1 / 5$ y $1 / 40$ del peso de varios de los taxones de megamamíferos extintos. Un factor relevante en esta discusión es la posibilidad de que la mayor parte del procesamiento de estos animales grandes se realizara en los sitios de matanza, por lo que en los sitios de procesamiento inicial como CT, tendríamos escasa proporción de huesos. Este fenómeno ha sido observado en repetidas oportunidades en contextos etnográficos/etnoarqueológicos (Bartram y Marean 1999; Bunn et al. 1988; O'Connell et al. 1990, entre otros).

En relación con el procesamiento, se observaron evidencias del mismo en los tres taxones, aunque con algunas variantes entre sí. En este sentido, tanto el guanaco como $L$. gracilis muestran evidencias tanto de corte como de percusión, mientras que $H$. paradoxa solo presenta evidencias de corte. Es destacable que la frecuencia de las marcas de corte y percusión no es muy elevada, dado que en ningún caso supera el $20 \%$ del conjunto. Algo similar ocurre en el caso de la termoalteración, que también presenta frecuencias que no exceden el $20 \%$ de la muestra. En este último caso también la intensidad de exposición al fuego parece haber sido más intensa en el guanaco y $L$. gracilis, dado que presentan todos los estadios de termoalteración, mientras que $H$. paradoxa solo muestra un quemado leve. De esta forma, para los tres taxones tanto el procesamiento como la termoalteración se registran en frecuencias relativamente bajas. No obstante, algunas diferencias en las variables tafonómicas (estadios más elevados de meteorización, frecuencias más altas de marcas de roedores, así como porcentajes más importantes de adherencias indeterminadas y tinción de manganeso) sugieren algunas diferencias en el caso de $H$. paradoxa. Ello, junto con una representación de partes esqueletarias acotada y las mencionadas escasas evidencias de procesamiento y termoalteración, permiten defender la idea de un tratamiento diferencial de $H$. paradoxa en comparación con los otros camélidos presentes en CT.

El análisis detallado de la distribución de marcas en la superficie de los huesos avala las bajas frecuencias registradas. De esta forma, la categoría de marcas más representada en cuanto a cantidad corresponde a pocas, y las mismas se encuentran preferentemente concentradas en las cercanías de las epífisis. Al compararlas con modificaciones de etiología conocida, que permite asignarles una funcionalidad específica, la mayor parte de las marcas corresponde a actividades de desarticulación, seguidas por aquellas de descarne de partes. Esto último es consistente con la hipótesis inicialmente propuesta para el sitio, en el que el mismo funcionaría como un lugar de procesamiento primario de presas cazadas a poca distancia del lugar (Paunero et al. 2015). Asimismo, aunque en frecuencias menores, al considerar otro tipo de huellas es posible mencionar que en el sitio también se dio el consumo de médula y se llevó a cabo la formatización de artefactos óseos.

Es importante contextualizar los datos presentados hasta aquí con información paleoambiental que tenemos disponible para el área en general y para el sitio en particular. Las evidencias de los patrones de temperatura, precipitación y reconstrucción de las comunidades vegetacionales de la Patagonia indica que hacia fines del Pleistoceno el área poseía un clima más frío que en la actualidad, con baja precipitación lo que traía aparejado el establecimiento de una estepa fría (De Melo França et al. 2015; Hernández et al. 2019). En términos generales, la tendencia al establecimiento de condiciones postglaciales fue interrumpida entre 14400 y 12700 años AP por la denominada Inversión Fría Antártica (ACR por sus siglas en inglés, ver Pedro et al. 2016; WAIS Divide Project Members 2015). Hacia los 12600 años AP se resume el progresivo calentamiento postglacial hasta alcanzar un pico alrededor de los 11400 años AP (Moreno et al. 2009; Sugden et al. 2005). Estos registros, que indican condiciones fluctuantes para los ambientes de finales del Pleistoceno en el área, muestran alto grado de acuerdo con las condiciones inferidas a partir de la Meseta Central. De esta 
forma, la información paleoecológica registrada por De Porras (2010) para CT indica un cambio desde condiciones más frías que las actuales hacia mayor temperatura durante el momento de conformación del componente inferior. Esto se refleja en particular en las comunidades vegetacionales de La María, que al igual que en sectores más al norte de la meseta como Los Toldos, entre los 12000 y 10250 años AP, indican la existencia de un predominio de una vegetación subarbustiva graminosa con predominio de Ephedra frustillata y Nassauvia. Asimismo, aunque en otros sectores como en Piedra Museo los análisis polínicos del nivel inferior del sitio Alero El Puesto 1 (AEP1) indican un predominio de Asteraceae y Poaceae, con bajos valores de Nassauvia y Ephedra, se señala igualmente la existencia de condiciones climáticas similares a las anteriormente descritas: frías y áridas (Borromei 2003). Esto está indicando para el componente inferior de CT un ambiente con mayor presencia de pasturas, consistente con la presencia de especies de camélidos como Lama gracilis y caballos extintos. Esto es especialmente relevante si se corrobora la similitud de $L$. gracilis con la vicuña (Weinstock et al. 2009), que presenta una adaptación hacia un consumo más elevado de pastos que el guanaco. Esto a su vez permite plantear la interesante pregunta de cómo particionaban el ambiente las tres especies de camélidos, o alternativamente si competían por los mismos recursos. La respuesta es posiblemente una de las claves que contribuya a explicar la extinción de los camélidos pleistocénicos, entre otras especies.
A futuro planteamos la necesidad de profundizar con análisis de isótopos estables de estos taxones, así como análisis de micro y mesodesgaste para determinar la partición de nicho entre especies con aparentemente similares requerimientos dietéticos. Por otra parte, creemos necesario incorporar los otros taxones a los análisis aquí presentados, para lograr una comprensión del ambiente ecológico en el que se desarrolló el primer poblamiento de la Meseta Central de Santa Cruz. Como mencionamos en la introducción, CT presenta una cronología que ubica al componente inferior en momentos inmediatamente previos a la extinción de varias especies de megafauna Pleistocénica. Ello proporciona una ventana única para estudiar un proceso sobre el que todavía tenemos más interrogantes que certezas y que posiblemente tuvo consecuencias muy importante para las poblaciones humanas posteriores a la transición PleistocenoHoloceno (Moscardi et al. 2020; Valiza Davis 2020).

Agradecimientos: Agradecemos a la familia Behm por su invalorable ayuda durante nuestros trabajos en La María. Al equipo que participó en la excavación en las sucesivas y trabajosas campañas. A Iván Pérez, que leyó una versión preliminar del trabajo y realizó valiosos comentarios. A Fabiana Skarbun por la realización del mapa. Este trabajo fue financiado gracias a CONICET y a la UNLP. Finalmente, nuestro agradecimiento a tres revisores anónimos que contribuyeron a mejorar sustancialmente el manuscrito.

\section{Referencias Citadas}

Bartram, J.L. y C. Marean 1999. Explaining the "Klasies Pattern": Kua ethnoarchaeology, the Die Kelders Middle Stone Age archaeofauna, long bone fragmentation and carnivore ravaging. Journal of Archaeological Science 26:9-29.

Behrensmeyer, A. 1978. Taphonomic and ecologic information from bone weathering. Paleobiology 4:150-162.Binford, L.R 1981. Bones: Ancient Men and Modern Myths. Academic Press, New York

Bird, J.B. 1988. Travels and Archaeology in South Chile. University of Iowa Press, Iowa City.

Borrero, L.A. 1990. Taphonomy of guanaco bones in Tierra del Fuego. Quaternary Research 34:361-371.

Borrero, L.A. 1999. The prehistoric exploration and colonization of Fuego-Patagonia. Journal of World Prehistory 13:321-355.

Borrero, L.A. 2015. The process of human colonization of Southern South America: Migration, peopling and "The Archaeology of Place". Journal of Anthropological Archaeology 38:46-51.
Borrero, L.A. y N.V. Franco 1997. Early Patagonian huntergatherers: subsistence and technology. Journal of Anthropological Research 53:219-239.

Borrero, L.A. y F.M. Martin 2018. Archaeological discontinuity in Ultima Esperanza: A supra-regional overview. Quaternary International 473:290-305.

Borromei, A.M. 2003. Palynology at Piedra Museo Locality, Santa Cruz Province, Argentina. En Where the south winds blow. Ancient evidences for Paleo South Americans, editado por L.L. Miotti, M.C. Salemme y N. Flegenheimer, pp.113-120. Center for the Studies of the First Americans (CSFA) and Texas A \& M University Press, College Station.

Bunn, H.T., L. Bartram y E. Kroll 1988.Variability in bone assemblage formation from Hadza hunting, scavenging, and carcass processing. Journal of Anthropological Archaeology 7:412-457.

Cajal, J., E.P. Tonni y V. Tartarini 2010. The extinction of some South American camelids: the case of Lama (Vicugna) gracilis. Mastozoología Neotropical 17 (1):129-134. 
Casamiquela, R. y T.D. Dillehay 1989. Vertebrate and invertebrate faunal analysis. En Monte Verde: A Late Pleistocene Settlement in Chile I: Palaeoenvironment and Site Context, editado por T. Dillehay, pp. 205-25. Smithsonian Institution Press, Washington D.C.

Cueto, M. 2015. Análisis de los Procesos de Uso de Artefactos Líticos en Sociedades Cazadoras-Recolectoras. Ocupaciones Correspondientes a La Transición Pleistoceno/Holoceno, Meseta Central de Santa Cruz. British Archaeological Reports, Oxford.

Cueto, M. y D. Andreoni 2016. Análisis preliminar de la gestión de restos vegetales en ocupaciones del Pleistoceno final/Holoceno temprano en la Meseta Central de Santa Cruz. En Arqueología de Patagonia: De mar a mar, editado por F. Mena, pp. 430-440. CIEP / Nire Negro Ediciones, Santiago.

De Melo França, L., L. De Asevedo, M.A.T. Dantas, A. Bocchiglieri, L. dos Santos Avilla, R.P. Lopes y J.L.L da Silva 2015. Review of feeding ecology data of Late Pleistocene mammalian herbivores from South America and discussions on niche differentiation. Earth-Science Reviews 140:158-165.

De Nigris, M. y G. Mengoni Goñalons 2004. El guanaco como fuente de carne y grasas en Patagonia. En Contra Viento y Marea. Arqueología de Patagonia, editado por T. Civalero, P. Fernández y G. Guraieb, pp. 469-476. Instituto Nacional de Antropología y Pensamiento Latinoamericano, Buenos Aires.

De Porras, M.E. 2010. Dinámica de la Vegetación de la Meseta Central de Santa Cruz durante los Últimos 11.000 años: Forzantes Bióticos y Abióticos. Tesis doctoral inédita, Universidad Nacional de Mar del Plata, Mar del Plata.

Del Moral Sachetti, F., F. Lameda Camacaro, J.Vázquez y R. Zenteno Cárdenas 2011. Fuerza de mordedura y estrés mandibular en el jaguar (Panthera onça) durante la depredación de pecaríes (Artiodactyla: Tayassuidae) mediante la fractura de sus cráneos. Acta zoológica mexicana 27:757-776.

Elkin, D.C. 1995. Volume density of South American camelid skeletal parts. International Journal of Osteoarchaeology 5:29-37.

Fariña, R.A., S.F.Vizcaíno y G. De Iuliis 2013. Megafauna: Giant Beasts of Pleistocene South America. Indiana University Press, Bloomington.

Fidalgo, F., L.M.M. Guzmán, G.G. Politis, M.C. Salemme, E.P. Tonni, J.E. Carbonari, G.J. Gómez, R. Huarte y A.J. Figini 1986. Investigaciones arqueológicas en el Sitio 2 de Arroyo Seco [Pdo. de Tres Arroyos, Pcia. de Buenos Aires, República Argentina]. En New evidence for the Pleistocene Peopling of Americas, editado por A. Bryan, pp. 222-269. Center fot the Study of the First Americans, Cornvalis.

Fisher, J.W. 1995. Bone surface modifications in zooarchaeology. Journal of Archaeological Method and Theory 2 (1):7-68.

Frank, A.D. 2011. Tratamiento Térmico y Manejo del Fuego en Sociedades Cazadoras-Recolectoras de la Meseta Central de Santa Cruz. Tesis doctoral inédita, Facultad de Ciencias Naturales y Museo, Universidad Nacional de La Plata, La Plata.

Frank, A.D. 2012. Los fogones en la Meseta Central de Santa Cruz durante el Pleistoceno Final. Magallania 40:145-162.

García Añino, E. 2018. Estrategias de Consumo de Grandes Mamíferos a lo largo del Holoceno entre los CazadoresRecolectores de la Meseta Central de Santa Cruz. El Caso del Guanaco en el Sitio Cueva Maripe. Tesis doctoral inédita, Universidad Nacional de La Plata, La Plata.

Gasparini, G.M., M. De los Reyes, A. Francia, C.S. Scherer y D.G. Poiré 2017. The oldest record of Hemiauchenia Gervais and Ameghino (Mammalia, Cetartiodactyla) in South America: Comments about its paleobiogeographic and stratigraphic implications. Geobios 50:141-153.

Grayson, D. 1984. Quantitative Zooarchaeology. Academic Press, New York.

Hajduk, A.y M. Lezcano 2005. Un "nuevo-viejo" integrante del elenco de instrumentos óseos de Patagonia: los machacadores óseos. Magallania 33:63-80.

Hernández, F., C. Ríos y H.L. Perotto-Baldivieso 2019. Evolutionary history of herbivory in the Patagonian steppe: The role of climate, ancient megafauna, and guanaco. Quaternary Science Reviews 220:279-290.

Kaufmann, C. 2009. Estructura de Edad y Sexo en Guanaco. Estudios Actualísticos y Arqueológicos en Pampa y Patagonia. Sociedad Argentina de Antropología, Buenos Aires.

Labarca, R. 2020. The Camelids (Artiodactyla: Camelidae) and Equids (Perissodactyla: Equidae) from the Pilauco Site, Northwestern Chilean Patagonia. En Pilauco: A Late Pleistocene Archaeo-Paleontological Site, editado por M. Pino y G. Astorga, pp. 69-94. Springer, Cham.

Labarca, R. y A. Prieto 2009. Osteometría de Vicugna vicugna Molina, 1782 en el Pleistoceno final de Patagonia meridional chilena: Implicancias paleoecológicas y biogeográficas. Revista del Museo de Antropología 2:127-140.

Lyman, R. 1994. Vertebrate Taphonomy (Vol. XXVI). Cambridge University, Cambridge.

Marchionni, L. 2013. Comparación de las Distintas Historias Tafonómicas en Conjuntos Zooarqueológicos Provenientes de la Meseta Central De La Provincia de Santa Cruz. Tesis doctoral inédita, Facultad de Ciencias Naturales y Museo, Universidad Nacional de La Plata, La Plata.

Martin, P. S.1967. Prehistoric overkill. En Pleistocene Extinctions: The Search for a Cause, editado por P.S. Martin y H.E. Wright, pp. 75-120. Yale University Press, New Haven.

Martin, F. 2008. Bone-Crunching Felids at the End of the Pleistocene in Fuego-Patagonia, Chile. Journal of Taphonomy 6:337-372.

Martin, F. 2013. Tafonomia y Paleoecología de la Transición Pleistoceno-Holoceno en Fuego-Patagonia: Interacción entre Humanos y Carnívoros y su Importancia como Agentes en la Formación del Registro Fósil. Ediciones de la Universidad de Magallanes, Punta Arenas.

Martin, F., M. San Román y F. Morello 2011. Reevaluación tafonómica de la interacción entre cazadores-recolectores y fauna extinta a fines del Pleistoceno en Última Esperanza, Chile: Modelos de inserción y poblamiento. En Bosques, Montañas y Cazadores. Investigaciones Arqueológicas en Patagonia Meridional, editado por L. Borrero y K. Borrazzo, pp. 105-126. CONICET-IMHICIHU, Buenos Aires.

Martínez, G. y M. Gutiérrez 2011. Paso Otero 5: a summary of the interdisciplinary lines of evidence for reconstructing early human occupation and paleoenvironment in the Pampean region, Argentina. En Peuplements et préhistoire en Amériques, dirigido 
por D. Vialou, pp. 271-284. Editions du Comité des Travaux Historiques et Scientifiques, París.

Martínez, G.A., M.A. Gutiérrez, P.G. Messineo, C. A. Kaufmann y D. Rafusse 2016. Subsistence strategies in the Southern cone of South America during the late Pleistocene and early Holocene Argentina as a case study. Quaternary Science Reviews 144:55-65.

Martinic, M. 1996. La Cueva del Milodon (Ultima Esperanza, Patagonia chilena). Un siglo de descubrimientos y estudios referidos a la vida primitiva en el sur de America. Journal de la Société des Américanistes 82:311-323.

Massone, M. 2004. Los Cazadores Después del Hielo (Vol. VII). Santiago de Chile, Centro de Investigaciones Diego Barros Arana, Dirección de Bibliotecas, Archivos y Museos 173.

Méndez, C., O. Reyes, H. Velásquez, V. Trejo y A. Maldonado 2008. New evidence of human occupation during the PleistoceneHolocene transition in Central Patagonia. Current Research in the Pleistocene 25:16-18.

Menegaz, A., F. Goin y E.O. Jaureguizar 1989. Análisis morfológico y morfométrico multivariado de los representantes fósiles y vivientes del género Lama (Artiodactyla, Camelidae). Sus implicancias sistemáticas, biogeográficas, ecológicas y biocronológicas. Ameghiniana 26:153-172.

Mengoni Goñalons, G. 1981. Los materiales óseos de la cueva 2 de Los Toldos (expedición Menghin) y una aproximación a la metodología de análisis de restos faunísticos. RUNA, archivo para las ciencias del hombre 13 (1-2):59-68.

Mengoni Goñalons, G. 1987. Modificaciones culturales y animales en los huesos de los niveles inferiores del sitio Tres Arroyos I (Tierra del Fuego, Chile). Anales del Instituto de la Patagonia 17: 61-66.

Mengoni Goñalons, G. 1996. La domesticación de los camélidos sudamericanos y su anatomía económica. En Zooarqueología de Camélidos, editado por D. Elkin, G. Goñalons, D. Olivera, M. Reigadas y H. Yacobaccio, Vol. II, pp. 33-45. Buenos Aires.

Mengoni Goñalons, G. 1999. Cazadores de Guanacos de la Estepa Patagónica. Sociedad Argentina de Antropología, Buenos Aires.

Metcalf, J.L., C. Turney, R. Barnett, F. Martin, S.C. Bray, J.T Vilstrup, L. Orlando, R. Salas-Gismondi, D. Loponte, M. Medina, M. De Nigris, T. Civalero, P.M. Fernández, A. Gasco, V. Durán, K.L. Seymour, C. Otaola, A. Gil, R. Paunero, F.J. Prevosti, C.J.A Bradshaw, J.C. Wheeler, L. Borrero, J.J. Austin y A. Cooper 2016. Synergistic roles of climate warming and human occupation in Patagonian megafaunal extinctions during the Last Deglaciation. Science Advances 2 (6):e1501682.

Miotti, L.L. 1998 [1989]. Zooarqueología de la Meseta Central y Costa de la Provincia de Santa Cruz: Un Enfoque de las Estrategias Adaptativas Aborígenes y los Paleoambientes. Imprenta del Museo Municipal de Historia Natural de San Rafael, Mendoza.

Miotti, L.L. y M.C. Salemme 1999. Biodiversity, Taxonomic Richness and Generalist-Specialists economical systems in Pampa and Patagonia Regions, Southern South America. Quaternary International 53/54:53-68.

Miotti, L.L. y M.C. Salemme 2004. Poblamiento, movilidad y territorios entre las sociedades cazadoras-recolectoras de Patagonia. Complutum 15:177-206.
Mondini, M. 2003. Modificaciones óseas por carnívoros en la Puna argentina. Una mirada desde el presente a la formación del registro arqueofaunístico. Mundo de Antes 3:87-108.

Morales-Mejía, F., J. Arroyo Cabrales y O.J. Polaco 2010. Estudio comparativo de algunos elementos de las extremidades anteriores y posteriores y piezas dentales de puma (Puma concolor) y jaguar (Panthera onça). TIP Revista Especializada en Ciencias Químico-Biológicas 13:73-90.

Moreno, P.I., M.R. Kaplan, J.P. François, R. Villa-Martínez, C.M. Moy, C.R. Stern y P.W. Kubik 2009. Renewed glacial activity during the Antarctic cold reversal and persistence of cold conditions until $11.5 \mathrm{ka}$ in southwestern Patagonia. Geology 37 (4):375-378.

Morin, E. 2007. Fat composition, skeletal part selection and Nunamiut decision- making. Journal of Archaeological Science 34 (1):69-82

Morin, E. y E. Ready 2013. Foraging goals and transport decisions in Western Europe during the Paleolithic and Early Holocene. En Zooarchaeology and Modern Human Origins, editado por J.L. Clark y J.D. Speth, pp. 227-269. Springer, Dordrecht.

Moscardi, B., D.D. Rindel y S.I. Perez 2020. Human diet evolution in Patagonia was driven by the expansion of Lama guanicoe after megafaunal extinctions. Journal of Archaeological Science 115. https://doi.org/10.1016/j.jas.2020.105098.

Nami, H.G. y A. Menegaz 1991. Cueva del Medio: aportes para el conocimiento de la diversidad faunística hacia el PleistocenoHoloceno en la Patagonia Austral. Anales del Instituto de la Patagonia 20:117-132.

O'Connell, J.F., K. Hawkes y J. Blurton 1990. Reanalysis of large mammal body part transport among the Hadza. Journal of Archaeological Science 17:301-316.

Paunero, R.S. 2009. La colonización humana de la meseta central de Santa Cruz durante el Pleistoceno final: indicadores arqueológicos, referentes estratigráficos y nuevas evidencias. En Arqueología de Patagonia: una Mirada desde el Último Confín, editado por M.C. Salemme, F. Santiago, M. Álvarez, E. Piana, M. Vazquez y M.E. Mansur, Vol. 1, pp. 85-100. Editorial Utopías, Ushuaia.

Paunero, R.S., A.D. Frank, M. Cueto, F. Skarbun y C. Valiza Davis 2015. La ocupación pleistocénica de Cueva Túnel, Meseta Central de Santa Cruz: un espacio que reúne actividades en torno al procesamiento primario de presas. Atek $\mathrm{Na}$ 5:149-188.

Paunero, R.S., M.F. Paunero y D. Ramos 2010. Artefactos óseos en componentes del Pleistoceno final de las localidades La María y Cerro Tres Tetas, Santa Cruz, Argentina. En Zooarqueología a Principios del Siglo XXI: Aportes Teóricos, Metodológicos y Casos de Estudio, editado por M. Gutiérrez, M. De Nigris, P. Fernández, M. Giardina, A. Gil, A. Izeta, G. Neme y H. Yacobaccio, pp. 459-466. Ediciones del Espinillo, Buenos Aires.

Paunero, R.S., C. Valiza Davis, D. Rindel y A. Tessone 2017. La fauna pleistocénica: evidencias zooarqueológicas en la Meseta Central de Santa Cruz, los sitios de La María. Magallania 45:181-198.

Pedro, J.B., H.C, Bostock, C.M. Bitz, F. He, M.J. Vandergoes, E.J. Steig, B.M. Chase, C.E. Krause, S.O. Rasmussen, B.R. Markle y G. 
Cortese 2016. The spatial extent and dynamics of the Antarctic Cold Reversal. Nature Geoscience 9 (1):51-55.

Perez, S.I., M.B. Postillone, D. Rindel, D. Gobbo, P.N.Gonzalez y V. Bernal 2016. Peopling time, spatial occupation and demography of Late Pleistocene-Holocene human population from Patagonia. Quaternary International 425:214-223.

Prevosti, F.y F. Martin 2013. Paleoecology of the mammalian predator guild of Southern Patagonia during the latest Pleistocene: Ecomorphology, stable isotopes and taphonomy. Quaternary International 305:74-84.

Prieto, A. y J. Canto 1997. Presencia de un lamoide atipico en Cueva Lago Sofia 4 (Última Esperanza) y Tres Arroyos (Tierra del Fuego) Región de Magallanes, Chile. Anales del Instituto de la Patagonia 25:147-150.

Prieto, A. y R. Labarca 2011. Los sitios arqueológicos del Pleistoceno Final de Fuego-Patagonia austral: Nuevos hallazgos, nuevos problemas. Boletín de Arqueología PUCP 15:357-383.

Rindel, D. 2009. Arqueología de Momentos Tardíos en el Noroeste de la Provincia de Santa Cruz (Argentina): Una Perspectiva Faunística Tesis doctoral inédita, Facultad de Filosofía y Letras, Universidad de Buenos Aires, Buenos Aires.

Schaller, G.B. y J.M.C. Vasconcelos 1978. Jaguar predation on capybara. Zeitschrift Fur Saugetierkunde-International Journal of Mammalian Biology 43:296-301.

Scherer, C.S., J. Ferigolo, A.M. Ribeiro y C. Cartelle 2007. Contribution to the knowledge of Hemiauchenia paradoxa (Artiodactyla, Camelidae) from the Pleistocene of southern Brazil. Revista Brasileira de Paleontología 10:35-52.
Skarbun, F. 2011. La Organización Tecnológica en Grupos Cazadores Recolectores desde las Ocupaciones del Pleistoceno Final al Holoceno Tardío, en la Meseta Central de Santa Cruz, Patagonia. British Archaeological Reports, Oxford.

Skarbun, F., M. Cueto, A.D. Frank, A.D. y R.S. Paunero 2015. Producción, consumo y espacialidad en Cueva Túnel, Meseta Central de Santa Cruz, Argentina. Chungara Revista de Antropología Chilena 47 (1):85-99.

Sugden, D.E., M.J. Bentley, C.J. Fogwill, N.R.J. Hulton, R.D. McCulloch y R.S. Purves 2005. Late glacial glacier events in southernmost south america: a blend of 'northern' and 'southern' hemispheric climatic signals? Geografiska Annaler: Series A, Physical Geography 87 (2):273-288.

Valiza Davis, C. 2020. Zooarqueología de Cazadores-recolectores de la Meseta Central de Santa Cruz durante la Transición Pleistoceno-Holoceno. Tesis doctoral, Facultad de Ciencias Naturales y Museo, Universidad Nacional de La Plata, La Plata.

Valiza Davis, C. y A. Gasco 2019. Los camélidos de Cueva Tunel (Santa Cruz): desde la osteometría, hacia una diferenciación de especies. Revista Brasileira de Paleontología 22 (2):146-164.

WAIS Divide Project Members 2015. Precise interpolar phasing of abrupt climate change during the Last Ice Age. Nature 520 (7549):661-665.

Weinstock, J., B. Shapiro, A. Prieto, J.C. Marín, B.A. González, M. Thomas, P. Gilbert y E. Willerslev 2009. The Late Pleistocene distribution of vicuñas (Vicugna vicugna) and the "extinction" of the gracile llama ("Lama gracilis"): New molecular data. Quaternary Science Reviews 28:1369-1373. 